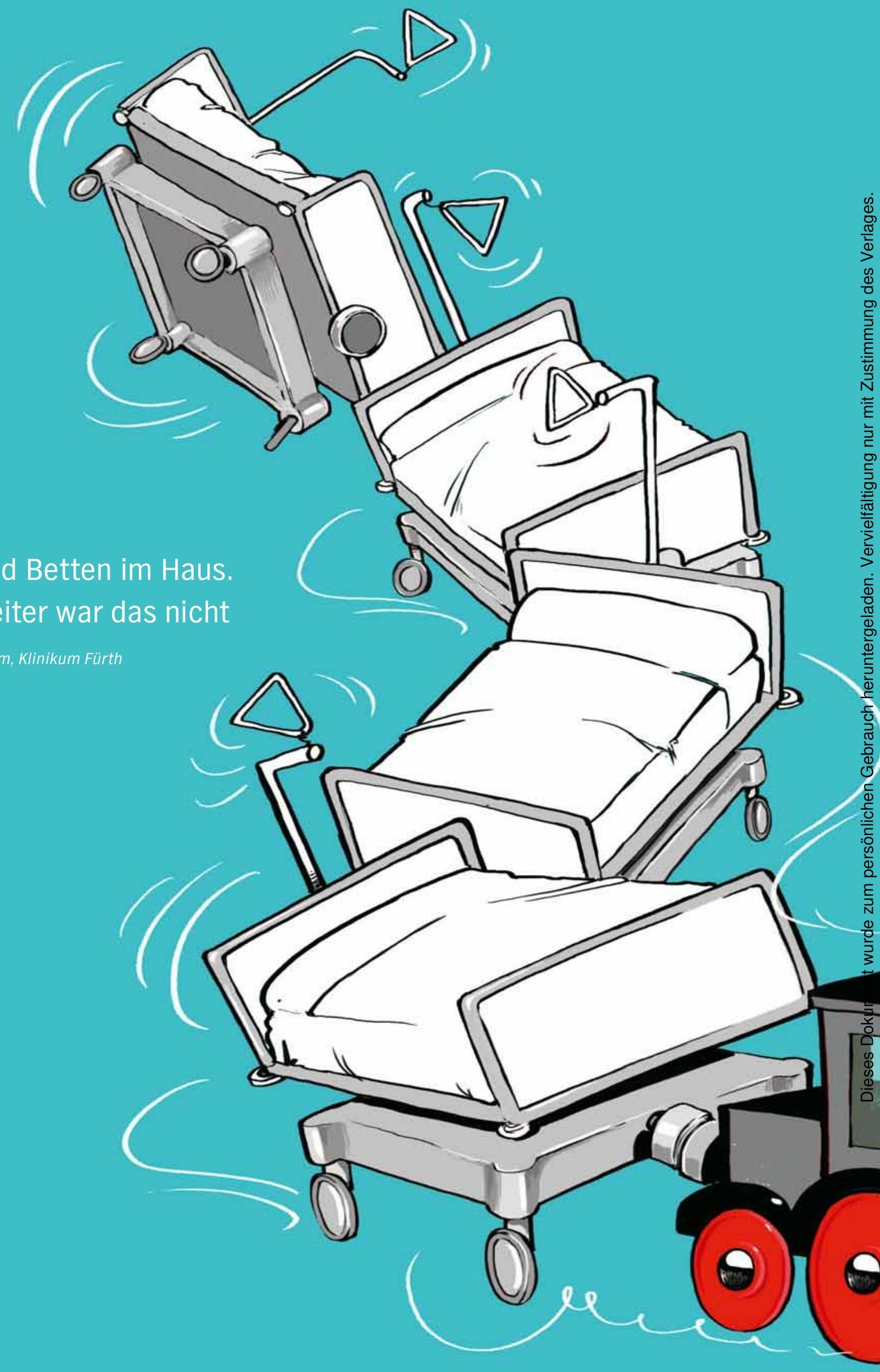




\section{Verschwendung auf Rädern}

In vielen Kliniken ist die Versorgung mit Betten intransparent und von Zufällen regiert. Nötig sind eine Neuorganisation des Bettenmanagements und klare Verantwortlichkeiten.

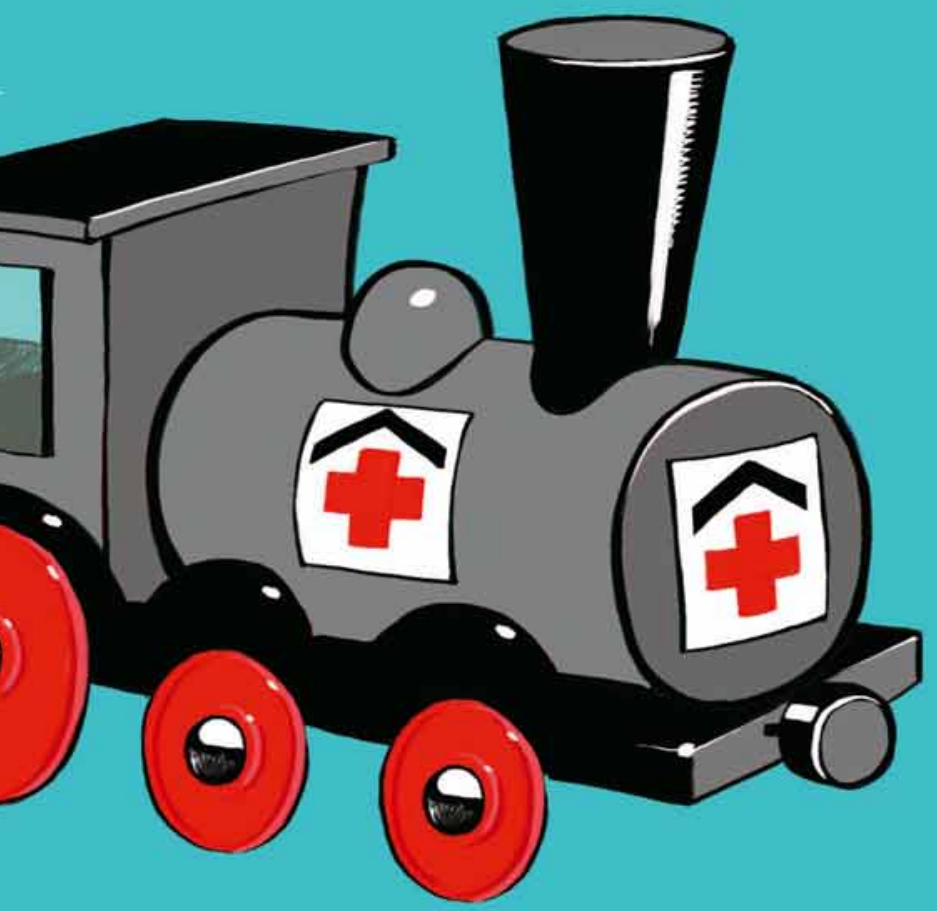

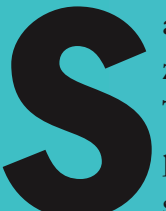
abine Salamon war ein Notfall, auch wenn sie zu Fuß ins Krankenhaus kam. Der Sturz von der Treppe war schon ein paar Wochen her, doch plötzlich wurden die Schmerzen schlimmer und schlimmer. Verdammtes Wochenende. Die Lehrerin wollte ein Röntgenbild, ein Schmerzmittel, Klarheit: Beckenbruch, ja oder nein? Und dann das Berliner Humboldt-Klinikum wieder verlassen, wie sie gekommen war: zu Fuß. Am Ende konnte sie das sogar. Und zwischendrin? „Da schob man mir ein Bett hin und sagte: Legen Sie sich erstmal hin!“

Einen Sitzplatz hätte Salamon als höflich und völlig ausreichend empfunden, und sie protestierte sogar - vergeblich. „Das ist hier so“, sagte jemand vom Personal. Salamon war verwundert: „Da gibt's keine Sitzplätze. Da gibt's nur Betten." Ihr Bett war frisch gemacht, was sonst? Und obwohl sie nur Patientin ist und keine Krankenhausexpertin, fielen ihr im Zuge dessen auch die 20 anderen Betten auf, die den Flur der Rettungsstelle füllten. „Hilflos gemacht und regelrecht genötigt" kam sie sich vor. Eine merkwürdige und enttäuschende Erfahrung war dies für eine Mutter, die dank der Blessuren von eigenen und Nachbarskindern das Klinikum in den vergangenen Jahren regelmäßig aufgesucht hatte und dessen medizinische Qualität ausdrücklich schätzt. Salamon wörtlich: „Die sind sonst richtig toll.“ 


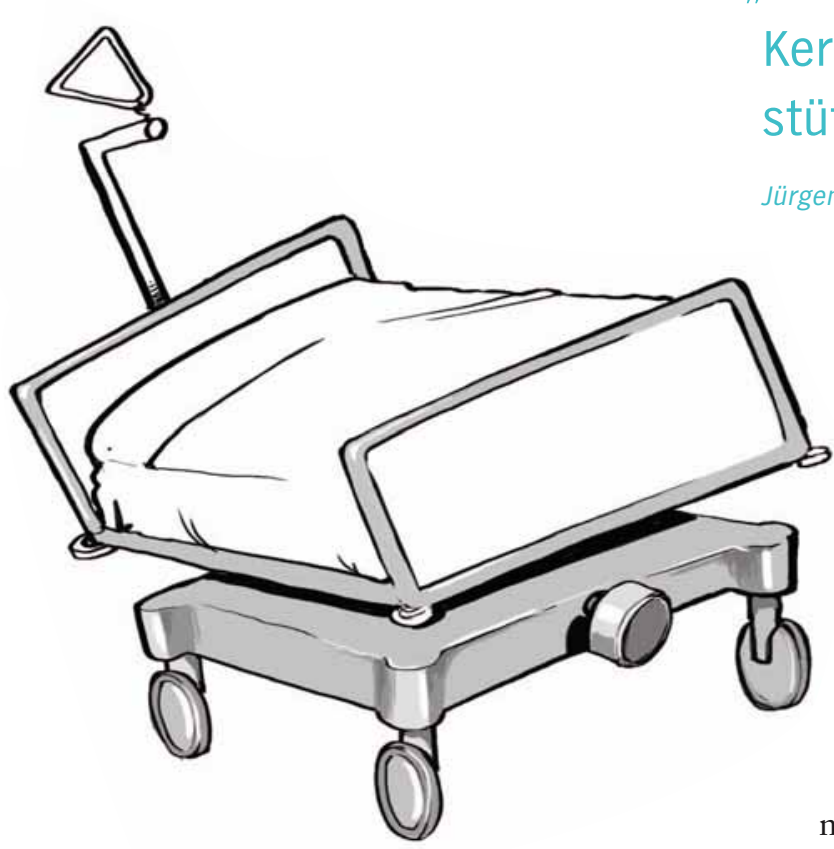

wirt mit dem Finger ganz und gar nicht nur auf andere.

Noch vor nicht all zu kurzer Zeit hatte er das Problem selbst. Sein Klinikum hatte im Jahr 2009 eine ganze Generation Krankenbetten ausgemustert und für rund drei Millionen Euro 600 neue Betten angeschafft. „Doch plötzlich war alles schlechter als vorher", erzählte Vitzithum. Objektiv und physisch waren genug Betten im Haus. „Nur: Das war für die Mitarbeiter nicht spürbar. Dieser relative und subjektive Mangel bestand darin, dass Mitarbeiter der Pflege Betten angefordert, aber nicht bekommen haben." Dies mündete am Ende in die Forderung nach noch mehr Betten - die dann an neuen und häufig unbekannten Stellen „als geheime Vorsorge gebunkert" worden beziehungsweise im Weg gestanden wären. Eine groteske ligsten." Vitzithum spricht von einer
Situation. In der Führungsetage schrillten die Alarmglocken. „Ja, die Bettenorganisation im Klinikum Fürth war verschwenderisch“, räumt Vitzithum offen ein - in vielerlei Punkten. (Siehe Kasten Seite 64: „So verschwenden Krankenhäuser Geld“). Die Klinikleitung entschloss sich dazu, die Blackbox zu knacken. In einem interdisziplinären Verbesserungsprojekt wurde das Bettenmanagement analysiert und neu organisiert, mit dem Ziel, die durchaus widersprüchlichen Ziele Verfügbarkeit, Fläche, Hygiene und Wirtschaftlichkeit unter einen Hut zu bekommen.

\section{Stretcher als Alternative}

Sabine Salamon hätte dieselbe Geschichte wie in Berlin auch in Fürth erleben können. Auch am städtischen Klinikum dort war in der Vergangenheit das Bett das einzige Lagerungsmittel in der Notaufnahme. Vitzithum nennt dies ironisch-trocken eine „aufnahme- und pflegeinduzierte „Blackbox“. Während im medizinischen Primärgeschäft Prozesse standardisiert und jeder Handgriff und jeder Cent erfasst und ausgewertet würden, passiere die Bettenversorgung eher, als dass sie bewusst gesteuert werde. „Moderne Krankenhäuser haben sehr komplexe Strukturen. Dadurch ist es oft schwer, die Betten im Umlauf und in der Aufbereitung zu überblicken und gleichzeitig die Kosten im Griff zu behalten“, sagt Christian Heister, Leiter des Bereichs Process beim Bettenhersteller Stiegelmeyer. „Einen echten Kümmerer auf Leitungsebene gibt es kaum“, kritisierte Vitzithum kürzlich bei einem Expertenforum zu diesem Thema auf der Fachmesse Medlogistica in Leipzig. Dabei zeigte der Betriebs-

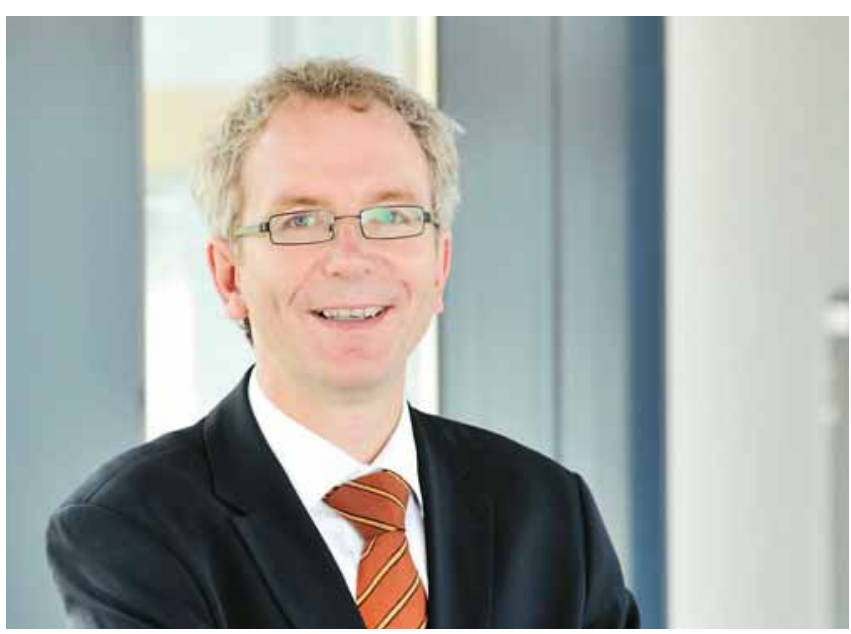

Martin Vitzithum: „Auch in unserer Notaufnahme war das Bett das einzige Lagerungsmittel. Der Patient war aufgeräumt. Die Pflege fühlte sich sicher. Der Arzt fühlte sich sicher." 
Bettlägrigkeit. Der Patient war aufgeräumt. Ärzte und Pflege fühlten sich sicher.“ Mit dem Verbesserungsprojekt gibt es hier mittlerweile alternative Lagerungsmöglichkeiten für Patienten, die keine Betten brauchen: Das Klinikum schaffte 60 Stretcher an, Transportliegen, die dank glatter Oberflächen bei ungleich geringerem Aufwand schnell und sicher gereinigt und desinfiziert werden können. Betten werden nur noch auf den Stationen eingesetzt. Die Flure sind jetzt frei. Es gibt klare Kommunikationsstrukturen. Die Bettenanforderung wird künftig IT-gestützt laufen und von einer Leitstelle koordiniert und kontrolliert. Der Gesamtprozess der Bettenlogistik wird damit auch dokumentiert und letztlich transparent gemacht - mit wertvollen Daten für kaufmännische und technische Leitung. Eine interne Verrechnung stationsbezogener Leistungen ist möglich. Das Krankenhaus kann externen Dienstleistern besser auf die Finger sehen und nachvollziehen, ob korrekt abgerechnet wurde oder nicht.

Mit dem obersten Ziel, die Verfügbarkeit spürbar zu verbessern, erproben das Klinikum Fürth, aber auch das Marienhospital Stuttgart, derzeit eine IT-gestützte Lösung, schneller und verbindlicher als das Telefon. Über den Stations-PC können Mitarbeiter mit wenigen Maus-Klicks eine Art Wunschliste ausfüllen - neues Bett bringen, schmutziges abholen, Patienten auf eine andere Station verlegen, Zubehör anfordern wie Aufrichter oder Dekubitusmatratzen. Das System wählt automatisch den richtigen Auftragnehmer aus und informiert ihn (Hol-/Bringdienst, Bettenzentrale, Reinigungsteam). „Wichtig ist, dass der Auftraggeber nicht mehr langwierig herumtelefonieren muss“, sagt Swen Walter, Gesamtvertriebsleiter beim Softwareanbieter Orgacard. „In dem Moment, wo er den Auftrag

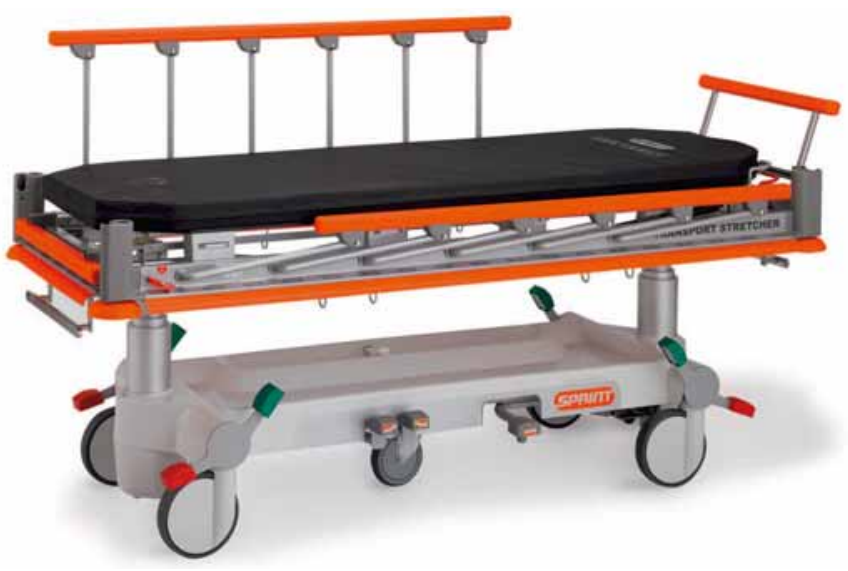

Bettenfreie Notaufnahme: Als Alternative für die zeitlich begrenzte Unterbringung von Patienten hat das Klinikum Fürth Transportliegen angeschafft. Bettenschlangen auf den Fluren sind hier Vergangenheit.

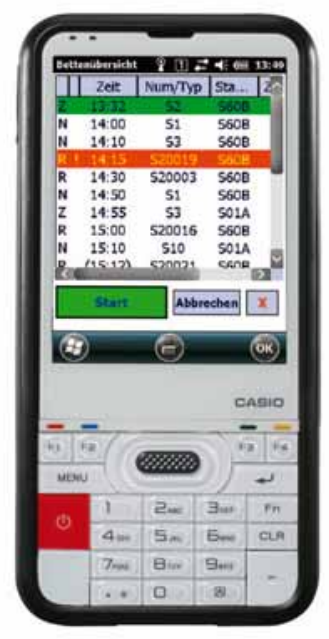

Bett auf Knopfdruck: Die Nutzung spezieller Software auf dem Stations-PC oder mobilen Endgeräten kann eine Lösung sein, um Bestellung, Transport oder Aufbereitung von Patientenbetten verbindlicher und professioneller zu organisieren und Transparenz in das Logistikgeschehen rund um das Bett zu bringen.

abschickt, ist er aus der Verantwortung und hat den Rücken frei. Die Arbeit macht dann das System.“ Für eine Erfassung und Auftragsvergabe auch unmittelbar am Bett hat das Klinikum Fürth testweise 15 Industrie-PDAs angeschafft: handliche mobile Endgeräte ähnlich wie Smartphones, nur viel robuster, für etwa 900 Euro pro Stück. Sie überleben es, wenn man sie einmal fallen lässt. Sie sind wischdesinfizierbar. Ihre Akkulaufzeiten halten viele Schichten
Klinikdienst durch. Bei Häusern mit Stationshilfen oder mobilen Reinigungsteams, also Bettenaufbereitung in den Zimmern, ist der PDA zugleich Auftragsempfangsgerät. Die Aufträge werden nach den Prioritäten zeitliche Dringlichkeit und räumliche Nähe sortiert. Sie können systematisch abgearbeitet werden, bei möglichst kurzen Wegen und möglichst geringem Zeitaufwand. Ist ein Job erledigt, wird dies vom Servicepersonal quittiert. Der Auftraggeber erhält eine Bestätigung. Swen Walter äußert Verständnis für die Mitarbeiter der Pflege: „Wenn ein Patient kommt, braucht er ein Bett. Und wenn ich als Stationsmitarbeiter zwei Stunden auf ein Bett warten muss, ist klar, dass ich horte. All das kann ich nur abstellen, wenn ich es schaffe, das Vertrauen wiederherzustellen, dass Betten auch zuverlässig geliefert werden.“

Um das Bettenmanagement von Grund auf zur reformieren und das richtige Bett zur richtigen Zeit am richtigen Ort zu haben - beim Patienten, in der Aufbereitung, in der Technik -, müssen sich Krankenhäuser die richtigen Fragen stellen und die richtigen Antworten geben. Wie viele Betten brauche ich wirklich? Was läuft gut, was schlecht bei mir? Welche Bettenaufbereitung ist für mich die beste? Eine besonders heikle Frage, auf die es die schnelle, einfache Antwort 


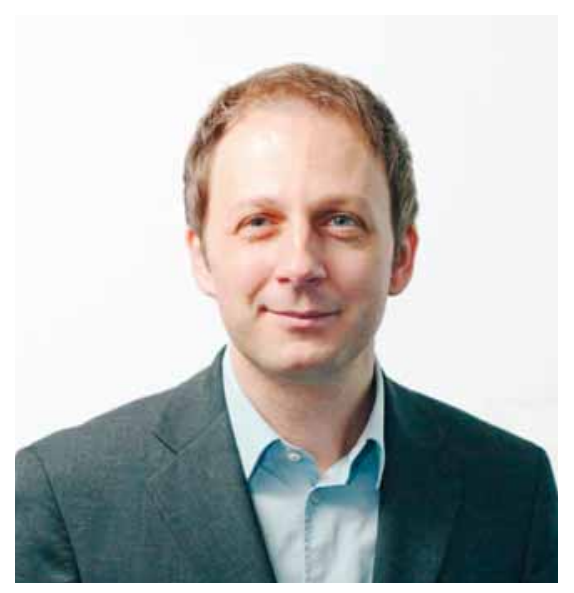

Jürgen Gerstetter, Marienhospital Stuttgart: „Krankenhäuser haben wegen kürzerer Verweildauern einen viel größeren Durchlauf."

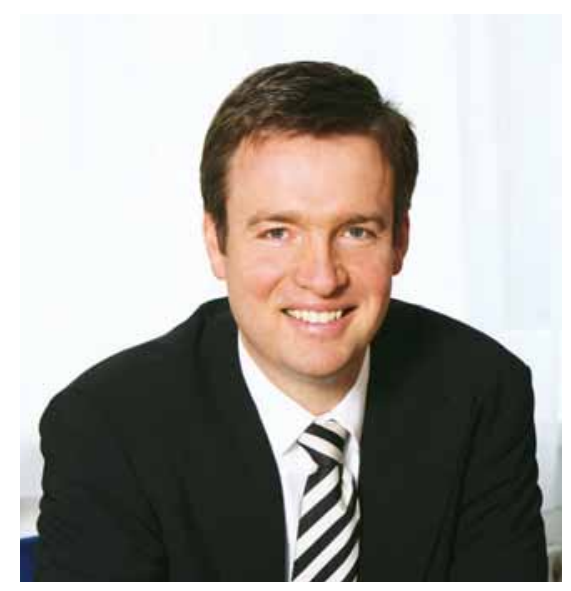

Andreas Hartung, Sana Kliniken: „Wir doktern lieber nicht an Symptomen herum. Entscheidend ist das Defizit in der Organisation."

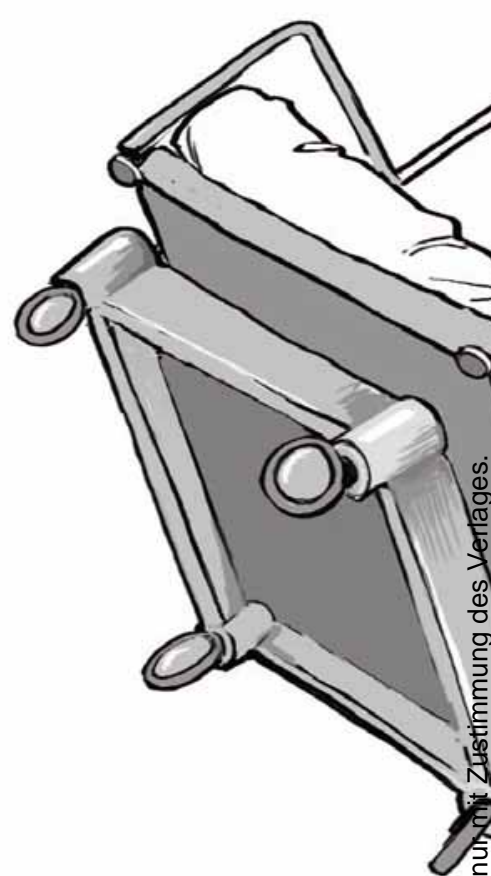

DESORGANISATION BEI DER BETTENVERSORGUNG

\section{So verschwenden Krankenhäuser Geld}

1. Gehfähige Patienten werden in Betten temporär "aufgeräumt“, unnötige Aufbereitungen sind die Folge,

2. Unnötige Bettentransporte behindern den Aufzugverkehr, verursachen Wartezeit und bremsen andere Abläufe aus,

3. Unnötige Transporte binden Personalkapazitäten beim Hol-undBringdienst und kosten Wegezeit,

4. Betten werden durch höhere Frequenz von Transporten stärker strapaziert/beschädigt, vermeidbare Reparaturkosten fallen an,

5. Wände/Türen/Inventar werden stärker strapaziert/beschädigt, Renovierungs-/Reparaturkosten fallen an,

6. Flure,Stellflächen und Fluchtwege werden durch benutzte oder auf Vorrat gehaltene Betten blockiert,

7. Numerischer Bettenbestand größer als nötig, unnötige Investitionskosten.

nicht gibt. Jedes der 2.000 deutschen Krankenhäuser ist unterschiedlich strukturiert, organisiert und finanziell ausgestattet und muss für sich das passende Konzept finden. Um diese Fragen systematisch und fundiert zu beantworten, bietet etwa der Hersteller Stiegelmeyer ein Beratungsprogramm für die bestmögliche Bettenlogistik im Krankenhaus an: mit einer Ist-Analyse und Lösungsvorschlägen; mit In-House-Schulungen für den richtigen Umgang mit modernen, elektromotorischen Betten, die dank ihres technischen Innenlebens immer mehr können, aber schwieriger zu bedienen, aufzubereiten und zu reparieren sind („Bettenführerschein“); und einem Monitoring für eine spätere erfolgreiche Umsetzung. Diese Beratungsleistung erfolgt laut Stiegelmeyer unabhängig davon, von welchem Hersteller die Betten in einem Haus stammen.

\section{Brennpunkt Aufnahme}

„Wir doktern lieber nicht an Symptomen herum. Entscheidend ist das Defizit in der Organisation": Andreas Hartung ist Generalbevollmächtigter Region Südost der Sana Kliniken und gehört zu den treibenden Kräften eines etwas anderen Modellprojekts, das aktuell in 12 der 50 Einzelhäusern läuft, die die private Klinikkette betreibt. Nicht so sehr die Verflüssigung der Logistik steht hier im Vordergrund, sondern vor allem die Steuerung der Patientenströme bei Aufnahme, Entlassung und in der Notaufnahme, die als kritische Größen gelten. „Die Elektivaufnahme haben wir - medizinisch wie administrativ - organisiert wie in einer Großpraxis“, sagt Hartung. Das heißt: zentral und in Abkehr von der konventionellen, säulenartigen Organisationsform. Als zweites Novum führte Sana ein zentrales Belegungsmanagement ein. Und drittens glättete der Konzern den Betrieb in der Notaufnahme. Viele der Sana-Häuser liegen in ländlichen Regionen. Die Notaufnahmen dort würden, insbesondere an Wochenenden, regelmäßig mit Patienten „geflutet“. Sana hat deshalb in den Modellhäusern die Manchester-Triage eingeführt, bei der die Patienten nach Dringlich- 

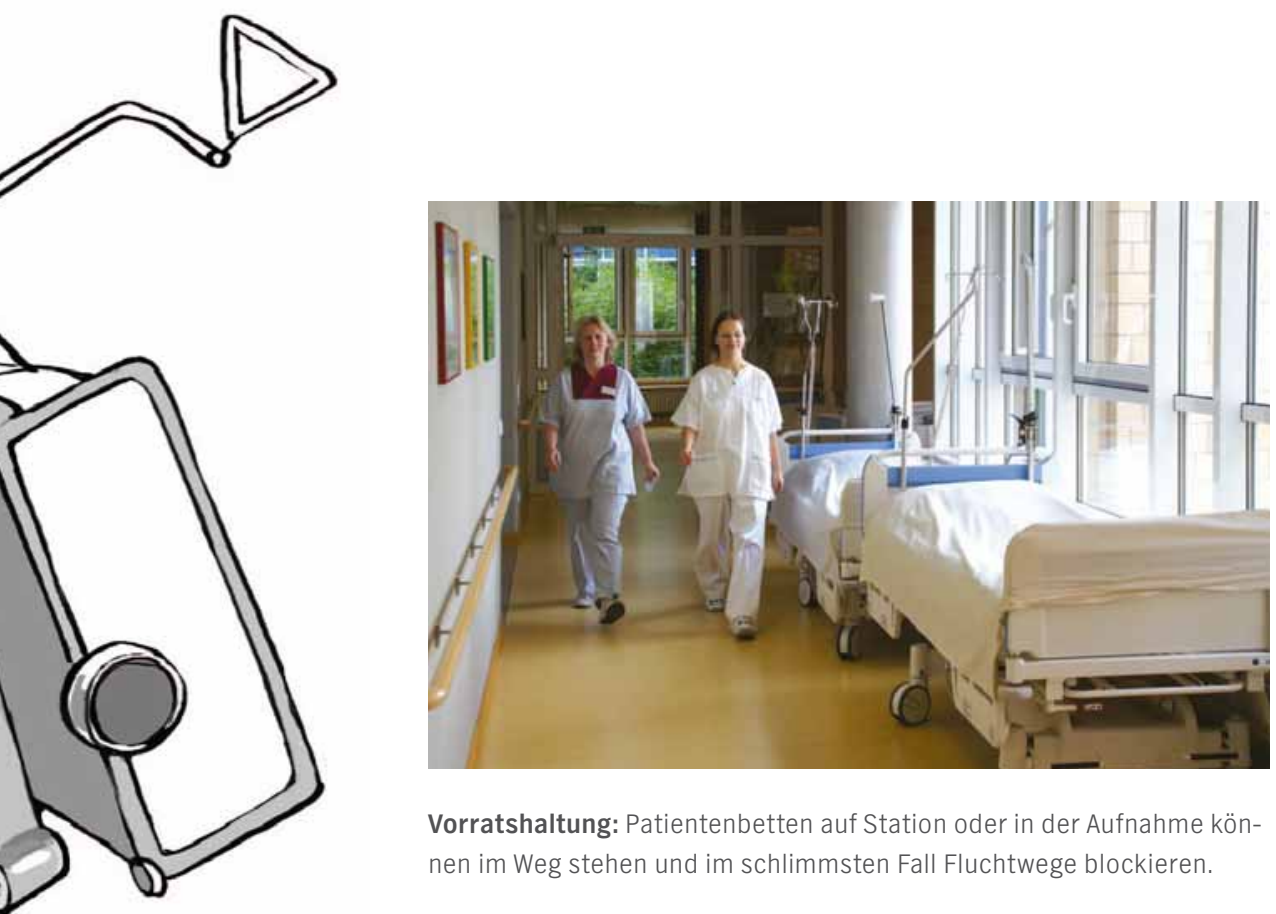

Vorratshaltung: Patientenbetten auf Station oder in der Aufnahme können im Weg stehen und im schlimmsten Fall Fluchtwege blockieren.

keiten sortiert werden - und immer möglichst schnell weitervermittelt.

Aus naheliegenden Gründen haben Krankenhäuser ihren Fokus bisher zu allererst auf die Verbesserung der Prozesse in ihrem Kerngeschäft Medizin mit den OPs und Funktions- und Diagnostikbereichen gerichtet. Aber auf die Dauer genügt das nicht, meinen Experten. Die Quasi-Halbierung der Liegedauer in den vergangenen 20 Jahren und der Anstieg der stationären Fälle haben auch die Frequenz von Bettentransporten und -aufbereitung in die Höhe schießen lassen. Die Infrastruktur vieler Krankenhäuser kam da nicht hinterher. „Viele Krankenhäuser haben das lange unterschätzt", sagt Swen Walter von Orgacard. „Auch war der Leidensdruck offenbar nicht groß genug. “ Langsam erwache aber das Bewusstsein dafür, dass auch in den Sekundärprozessen jenseits der Medizin viel Geld stecke. „Viele Häuser sind mit dem ersten Schritt durch", beobachtet der KrankenhausIT-Fachmann. „Jetzt kümmern sie sich um den zweiten." Und beim Bettenmanagement scheint dies dringend geraten, weil Nachlässigkeit hier, etwa durch Wartezeiten vor Aufzügen oder im OP, bei vielen anderen Prozessen und nicht zuletzt beim interessanten medizinischen Kerngeschäft Sand ins Getriebe bringt.

\section{Kostbare Ressource}

„Krankenhäuser haben aufgrund der verkürzten Verweildauer einen viel größeren Durchlauf heute“, sagt Jürgen Gerstetter, Pflegedirektor am Stuttgarter Marienhospital. „Wenn sich die pflegerischen und medizinischen Kernprozesse immer mehr verdichten, dann müssen auch die unterstützenden Prozesse reibungslos laufen, sonst hat dies einschränkende Auswirkungen auf die Qualität der Patientenversorgung.“ ” Außerdem sind Betten Investitionsgüter, die immer empfindlicher werden, weil sie immer komfortabler und intelligenter werden. Deshalb ist es klug und letztlich auch rentabel, bewusst und pfleglich mit ihnen umzugehen und sie als das zu behandeln, was sie sind: eine kostbare Ressource. Seit das Betten-Verkehrsaufkommen hier abgenommen hat, registriert das Klinikum Fürth erste Erfolge. Weniger Schäden an Wänden und Türen. Weniger Reparaturen an Betten. Und die Patienten, die Kunden, werden medizinisch adäquat gelagert und im Idealfall auch so, wie sie es angenehm finden. Damit ชั้ erübrigt sich für sie die Frage, die sich Sabine Salamon am Ende stellte: ob sie ฐั๊ wiederkommen würde oder nicht. ㄸ

Adalbert Zehnder
- Dokumentation

- Reinigung und Desinfektion

- Packen und Sterilisation

- Logistik und Lagerung
- Service und Validierung

- Beratung und Realisierung
- Schulung des ZSVA-Personals

- Hygieneorientierte Ablaufoptimierung 